\title{
Transgenic Expression of Human Thrombomodulin Inhibits HMGB1-Induced Porcine Aortic Endothelial Cell Activation
}

Anjan K. Bongoni, PhD, ${ }^{1}$ Nikolai Klymiuk, PhD, ${ }^{2}$ Eckhard Wolf, DVM, ${ }^{2}$ David Ayares, PhD, ${ }^{3}$ Robert Rieben, PhD, ${ }^{4}$ and Peter J. Cowan, $\mathrm{PhD}^{1,5}$

Background. Transgenic expression of human thrombomodulin (hTBM), which has the potential to solve the problem of coagulation dysregulation in pig-to-primate xenotransplantation, may have additional benefits by neutralizing the proinflammatory cytokine high-mobility group box 1 (HMGB1). The aim of this study was to investigate HMGB1-mediated effects on porcine aortic endothelial cells (PAEC) from wild-type (WT) and hTBM transgenic pigs. Methods. Porcine aortic endothelial cells were treated with HMGB1, human (h)TNFa or lipopolysaccharide (LPS). Procoagulant and proinflammatory responses were assessed by measuring expression of cell surface markers (adhesion molecules, fibrinogen-like protein 2, plasminogen activator inhibitor (PAI)-1), secretion of porcine cytokines and chemokines (HMGB1, TNFa, IL-8, monocyte chemotactic protein-1), and formation of PAl1/tissue plasminogen activator complexes. Thrombin-mediated degradation of HMGB1 in the presence of PAEC was examined by Western blot and functional assay. Results. High-mobility group box 1 potently activated WT PAEC, increasing the expression of E-selectin, vascular cell adhesion molecule-1, intercellular adhesion molecule-1, fibrinogen-like protein 2, and PAl-1, the secretion of TNFa, IL-8, and monocyte chemotactic protein-1 and the formation of PAl-1/tissue plasminogen activator complexes. Human TNFa- or LPS-induced activation of WT PAEC was inhibited by treatment with rabbit anti-HMGB1 antibody. Transgenic expression of hTBM significantly reduced the activation of PAEC by HMGB1 or hTNFa, and significantly enhanced thrombininduced HMGB1 cleavage. Chemically induced shedding of the lectin-like domain of TBM resulted in significantly increased HMGB1-induced PAEC activation. Conclusions. High-mobility group box 1 exerts powerful proinflammatory and procoagulant effects on WT PAEC, and appears to be an important downstream mediator for the actions of hTNFa and LPS. Human thrombomodulin transgenic PAECs are less sensitive to activation by either HMGB1 or hTNFa, an effect that appears to be dependent on the lectin-like domain of TBM.

(Transplantation 2016;100: 1871-1879)

ig-to-human xenotransplantation offers a potential solution to the chronic shortage of human donor organs. However, porcine xenografts induce a powerful immune response, in which coagulation and inflammation play an important role. Genetically modified pigs expressing human complement regulatory proteins on the $\alpha 1,3$-galactosyltransferase gene knockout (GTKO) background have been developed to address the initial barrier of hyperacute rejection. However, coagulation dysregulation due to immune responses and cross-species

\footnotetext{
Received 20 December 2015. Revision received 12 January 2016.

Accepted 18 January 2016.

${ }^{1}$ Immunology Research Centre, St. Vincent's Hospital Melbourne, Victoria, Australia. 2 Institute of Molecular Animal Breeding and Biotechnology, Ludwig-Maximilian University, Munich, Germany.

${ }^{3}$ Revivicor, Inc., Blacksburg, VA.

${ }^{4}$ Department of Clinical Research, University of Bern, Bern, Switzerland.
}

${ }^{5}$ Department of Medicine, University of Melbourne, Melbourne, Victoria, Australia.

These studies were supported by grants from the Swiss National Science Foundation (SNSF) (Fellowship: P2BEP3_155459 to AKB and grant 320030_156193 to RR), the National Health \& Medical Research Council of Australia and - in part - by the German Research Council (CRC TR127).

The authors declare no conflicts of interest.

A.K.B. participated in the research design, writing of the article, performance of the research, and data analysis. N.K., E.W. participated in production of molecular incompatibilities has emerged as a major obstacle to longer term xenograft survival. ${ }^{1}$

The thrombomodulin (TBM)/protein C pathway is a critical regulator of coagulation and inflammation within the microvasculature. Thrombomodulin is an integral endothelial membrane protein that alters the specificity of thrombin from procoagulant and proinflammatory substrates to protein C, which in its activated form inhibits coagulation and inflammation. However, this key regulatory mechanism is compromised in xenotransplantation because pig TBM binds human

hTBM and galactosyltransferase gene-knockout/hCD46/hTBM pigs. D.A. provided primary cells from hCD46 transgenic pigs for nuclear transfer experiments. R.R. provided scientific support and reagents and participated in the critical revision of the article. P.J.C. participated in the concept and design of the study, performance of experiments, analyzing the data, writing the manuscript, and carried the main responsibility for the study.

Correspondence: Peter Cowan, Immunology Research Centre, St. Vincent's Hospital Melbourne, PO Box 2900, Fitzroy 3065, Victoria, Australia. (peter. cowan@svha.org.au).

Supplemental digital content (SDC) is available for this article. Direct URL citations appear in the printed text, and links to the digital files are provided in the HTML text of this article on the journal's Web site (www.transplantjournal.com).

Copyright (C) 2016 Wolters Kluwer Health, Inc. All rights reserved.

ISSN: 0041-1337/16/10009-1871

DOI: $10.1097 / T P .0000000000001188$ 
thrombin but is a poor cofactor for the activation of human protein $\mathrm{C}$, with only less than $10 \%$ of the activity of human TBM (hTBM). ${ }^{2}$ Transgenic expression of hTBM is a promising approach to overcome this molecular incompatibility. Transgenic hTBM on porcine aortic endothelial cells (PAEC) prolonged the clotting time of human blood in vitro. ${ }^{3}$ In vivo, hTBM expression on cardiac xenografts was associated with a delay in the development of thrombotic microangiopathy and/or consumptive coagulopathy. ${ }^{4}$

In addition to its anticoagulant properties, TBM also attenuates inflammation through activated protein C-dependent and -independent mechanisms. The lectin-like domain of TBM inhibits complement activation and neutralizes endotoxin. ${ }^{5}$ It also sequesters the "danger signal" high-mobility group box 1 (HMGB1) and facilitates its cleavage by thrombin. High-mobility group box 1 is a highly conserved protein present in the nucleus and cytoplasm of almost all cell types (reviewed in Ito et $\mathrm{al}^{6}{ }^{6}$. High-mobility group box 1 serves a dual function as an intracellular DNA-binding protein, maintaining nucleosome structure and regulation of gene transcription, and as an extracellular signal of tissue damage. High-mobility group box 1 is passively released from necrotic or damaged cells or actively secreted by inflammatory cells, including activated monocytes, macrophages, neutrophils, and endothelial cells. ${ }^{7,8}$ Extracellular HMGB1 initiates potent innate immune responses by promoting chemotactic cell interactions, cytokine production, T cell activation, dendritic cell maturation, and coagulation activation, via its binding to pattern recognition receptors including the receptor for advanced glycation end-products and Tolllike receptors 2 (TLR2) and 4 (TLR4). ${ }^{8,9}$

Although initial findings demonstrated HMGB1 as a late mediator of sepsis, ${ }^{10}$ recent studies indicate that HMGB1 also plays a pivotal role in the pathogenesis of a range of inflammatory states ${ }^{11}$ including transplant rejection. ${ }^{12}$ Anti-HMGB1 treatment can significantly prolong cardiac xenograft survival in a rat-to-mouse model. ${ }^{13}$ However, HMGB1-mediated activation of porcine endothelial cells has not been explored, and the protective effect of hTBM against HMGB1's actions in this setting has not been examined. We therefore aimed to test HMGB1-mediated proinflammatory and procoagulant effects on PAEC and to assess the capacity of transgenic expression of hTBM on PAEC to neutralize HMGB1.

\section{MATERIALS AND METHODS}

\section{Cell Culture}

Porcine aortic endothelial cells were isolated from wildtype (WT), hTBM- or GTKO.hCD46. hTBM transgenic pig aorta as described previously. ${ }^{3,14}$ Porcine aortic endothelial cells were used between passages 2 and 8 in all experiments.

\section{Cell Enzyme Linked Immunosorbent Assay}

Confluent WT, hTBM, and GTKO.hCD46. hTBM PAEC grown in 96-well plates were treated with HMGB1 (HMGBiotech, Milan, Italy; 50-200 ng/mL) or human (h) TNF $\alpha$ (R\&D Systems, Minneapolis, MN; $10 \mathrm{ng} / \mathrm{mL}$ ) or lipopolysaccharide (LPS) from Escherichia coli 026: B6 (SigmaAldrich, Castle Hill, Australia; $100 \mathrm{ng} / \mathrm{mL}$ ) in the presence or absence of rabbit anti-HMGB1 antibody (Sigma-Aldrich) at $37^{\circ} \mathrm{C}$ for 4 to 24 hours. In some experiments, PAEC were pretreated with either human thrombin (Merck Millipore, Bayswater, Australia; $5 \mathrm{U} / \mathrm{mL}$ for 60 minutes) or lysophosphatidic acid (LPA) (10 $\mu \mathrm{M}$ for 10 minutes). After fixation with $3.7 \%$ formalin for 15 minutes and blocking with phosphatebuffered saline- $3 \%$ bovine serum albumin for 30 minutes at room temperature, cells were incubated with mouse antihuman E-selectin (fluorescein isothiocyanate [FITC] labeled; AbD Serotec, Oxford, UK), mouse antipig vascular cell adhesion molecule (VCAM)-1 (10.2C7, generously provided by D.O. Haskard [Department of Immunology, Royal Postgraduate Medical School, Hammersmith Hospital, London, UK]), mouse antihuman intercellular adhesion molecule (ICAM)-1 (FITC; AbD Serotec), rabbit antihuman fibrinogen like-2 (FGL2, Aviva Systems Biology, San Diego, CA), mouse antihuman plasminogen activator inhibitor-1 (PAI-1) (Hycult Biotech, Uden, The Netherlands), and rabbit antihuman tissue plasminogen activator (tPA) (Molecular Innovations, Novi, MI). Subsequently, biotin-conjugated rabbit anti-mouse IgG (Dako, Braeside, Australia) or goat antirabbit IgG (Dako) and alkaline phosphatase conjugated rabbit anti-FITC (Abcam, Melbourne, Australia) or alkaline phosphatase (AP)-streptavidin (GE Healthcare, Parramatta, Australia) were used. Finally, p-nitrophenyl phosphate substrate (SigmaAldrich) was added and color development was quantified using a FLUOstar Omega microplate reader.

\section{Enzyme Linked Immunosorbent Assay}

Fluid phase PAI-1/tPA complex was measured as an antifibrinolytic marker using mouse antihuman PAI-1 (Hycult Biotech) and biotinylated rabbit antihuman tPA (Molecular Innovations) antibodies. High-mobility group box 1 in culture supernatant was detected using an in-house developed enzyme linked immunosorbent assay (ELISA) with mouse antihuman HMGB1 and horseradish peroxidase-conjugated rabbit antihuman HMGB1 (Abcam) antibodies. Full-length HMGB1 protein (Abcam) was used as a standard. Streptavidin-AP and the appropriate substrate were used for color development.

Production of porcine cytokines (TNF $\alpha$, IL-8, and monocyte chemotactic protein [MCP]-1) by PAEC was measured in cell culture supernatants using specific porcine DuoSet kits (TNF $\alpha$ and IL-8, R\&D Systems) and MCP-1 ELISA kit (PeproTech, Rocky Hill, NJ) according to manufacturer's protocol.

\section{Western Blot}

Porcine aortic endothelial cells were pretreated with thrombin (Merck Millipore) for 30 minutes, followed by HMGB1 treatment for 30 minutes. Cells were lysed in radioimmunoprecipitation buffer (Sigma-Aldrich) with protease inhibitor cocktail (Sigma-Aldrich). Samples were electrophoresed on $4 \%$ to $20 \%$ mini-Protean TGX precast gels (Bio-Rad, Gladesville, Australia) and analyzed by Western blot. Membranes were blocked with 50\% Odyssey blocking buffer (LI-COR Biosciences, Lincoln, NE) and stained with anti-HMGB1 (Abcam). Thereafter, membranes were probed with the appropriate secondary antibodies conjugated to IRDye 680CW (LI-COR Biosciences). Membranes were scanned with a LI-COR near-infrared Odyssey scanner (LICOR Biosciences). Quantification of fluorescence intensity as raw integrated density was performed using Image J software, version 10.2 (National Institutes of Health). 

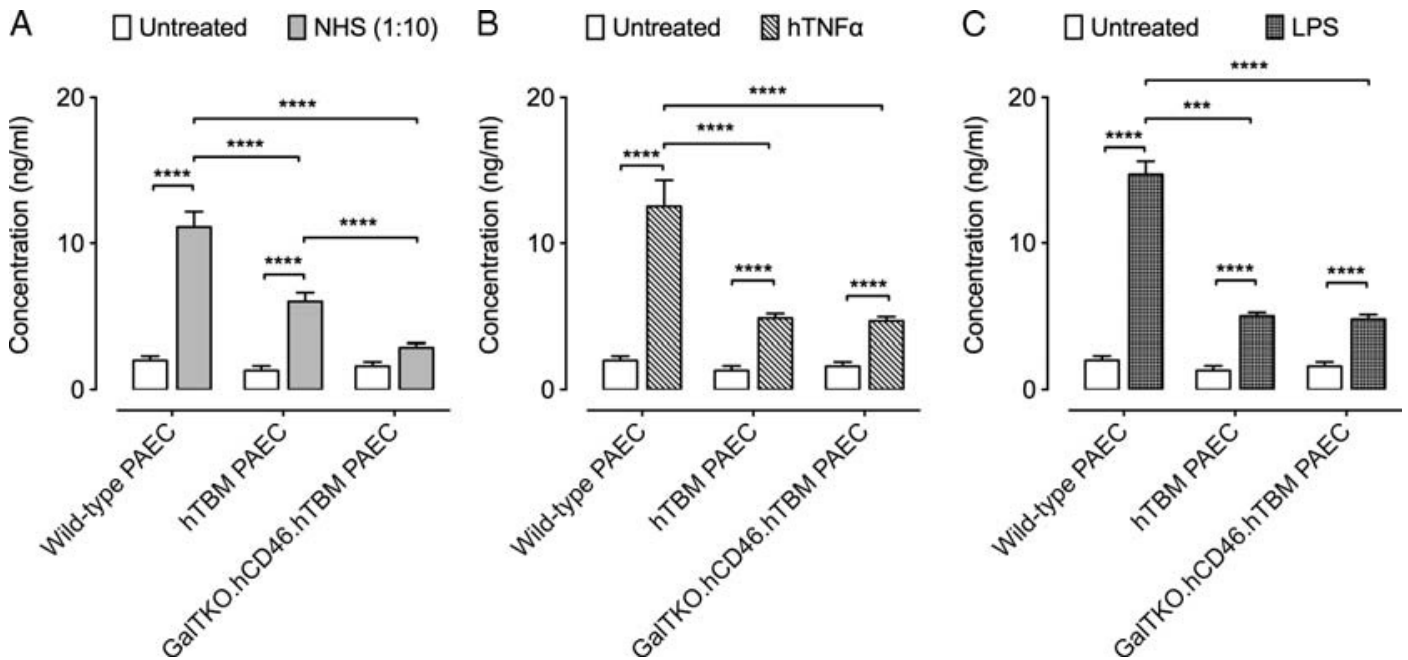

FIGURE 1. Effect of transgenic expression of hTBM on the NHS-, hTNFa- or LPS-induced release of HMGB1 from PAEC. Confluent WT, hTBM, and GTKO.hCD46.hTBM PAEC grown in 12-well plates were stimulated with (A) NHS (1:10) or (B) hTNFa (10 ng/mL) or (C) LPS $(100 \mathrm{ng} / \mathrm{mL})$ for 16 hours, and the release of HMGB1 in cell culture supernatants was measured by ELISA. Statistical analysis was done by 1 -way ANOVA testing with Bonferroni correction $\left({ }^{\star} P<0.05\right.$, $\left.{ }^{\star \star} P<0.01,{ }^{\star \star \star} P<0.001,{ }^{\star \star \star \star} P<0.0001\right)$. All results are shown as mean \pm SD of 3 independent experiments. ANOVA, analysis of variance.

\section{Immunofluorescence Staining}

Wild-type and hTBM PAEC grown on 8-well Lab-Tek chamber slides (Milian, Geneva, Switzerland) were pretreated with LPA (10 $\mu \mathrm{M}$ for 10 minutes) and incubated with HMGB1 or hTNF $\alpha$ for 16 hours. After this, cells were incubated with mouse antihuman TBM (clone D-3; Santa Cruz Biotechnology, Geneva, Switzerland; and clone 141C01; Lifespan Biosciences, Seattle, WA) and rat anti-pig CD31 (R\&D Systems) for 60 minutes. Thereafter, cells were exposed to goat anti-rat IgG-Alexa594, and goat anti-mouse IgGAlexa488 (Molecular Probes). Nuclei were stained using 4',6diamidino-2-phenylindole (DAPI, Boehringer Ingelheim, North Ryde, Australia). The slides were analyzed using a Nikon A1R confocal microscope.

\section{Statistical Analysis}

Data are shown as mean \pm standard deviation. Statistical analysis was performed using GraphPad Prism 6 (GraphPad Software, San Diego, CA). Significance was tested using Student $t$ test and 1-way analysis of variance with Bonferroni correction $(* P \leq 0.05, * * P \leq 0.01, * * P \leq 0.001$, $* * * P \leq 0.0001)$.

\section{RESULTS}

\section{Human Serum, hTNF $\alpha$, and LPS Induce the Release of HMGB1 From PAEC}

Wild type, hTBM or GTKO.hCD46.hTBM PAEC were stimulated for 16 hours with normal human serum (NHS, 1:10), hTNF $\alpha(10 \mathrm{ng} / \mathrm{mL})$ or LPS (100 ng/mL), and analyzed for HMGB1 release in culture supernatants. As shown in Figure 1, a low amount of HMGB1 was detected in supernatants of all untreated PAEC $(1.3 \pm 0.3$ to $2.0 \pm 0.3 \mathrm{ng} / \mathrm{mL})$. Treatment of WT PAEC with NHS, hTNF $\alpha$, or LPS significantly increased HMGB1 levels, by 5.5-, 6-, and 7-fold, respectively. High-mobility group box 1 levels were also increased by each of the treatments of hTBM PAEC, and by hTNF $\alpha$ or LPS treatment of GTKO.hCD46.hTBM PAEC. However, the rise in HMGB1 under all conditions was significantly blunted in the transgenic cells compared with WT cells, suggesting that elevated TBM expression increased sequestration of HMGB1 and/or reduced activation of the PAEC. Interestingly, HMGB1 was not significantly increased by NHS treatment of GTKO.hCD46.hTBM PAEC, consistent with reduced xenoantibody binding, complement activation, and cellular activation.

\section{Anti-HMGB1 Treatment Inhibits hTNF $\alpha$ - and LPS-Induced Activation of WT PAEC}

To investigate the effects of HMGB1 inhibition on PAEC activation, WT PAEC were treated for 16 hours with hTNF $\alpha$ or LPS in the presence or absence of rabbit antihuman HMGB1 antibody (2 or $5 \mu \mathrm{g} / \mathrm{mL})$. Human TNF $\alpha$ and LPS significantly induced expression of the adhesion molecules E-selectin and VCAM-1, markers of endothelial cell activation, on WT PAEC (Figures 2A-B). However, anti-HMGB1 antibody treatment significantly reduced the upregulation of both markers, in a dose-dependent manner. Furthermore, anti-HMGB1 treatment significantly reduced the hTNF $\alpha$ and LPS-mediated secretion of porcine TNF $\alpha$, IL- 8 and MCP-1 (Figures 2C-E). These results suggest that HMGB1 release triggered by proinflammatory mediators is a central downstream amplifier of PAEC activation.

\section{Transgenic hTBM Inhibits HMGB1-Induced Activation of PAEC}

High-mobility group box 1 increased the expression of Eselectin, VCAM-1, and ICAM-1 on WT PAEC in a doseand time-dependent manner. Cotreatment with hTNF $\alpha$ and HMGB1 caused a further increase in activation over that observed with hTNF $\alpha$ treatment alone (Figure S1, SDC, http:// links.lww.com/TP/B262).

Incubation of confluent WT PAEC with HMGB1 $(100 \mathrm{ng} / \mathrm{mL}$, 16 hours) or hTNF $\alpha$ (10 ng/mL, positive control) resulted in significantly increased expression of E-selectin, VCAM-1, and ICAM-1 as compared with untreated PAEC. Stimulation of hTBM PAEC under the same conditions increased the expression of E-selectin and ICAM-1, but not VCAM-1. However, the rise in expression of these endothelial cell activation markers 
A

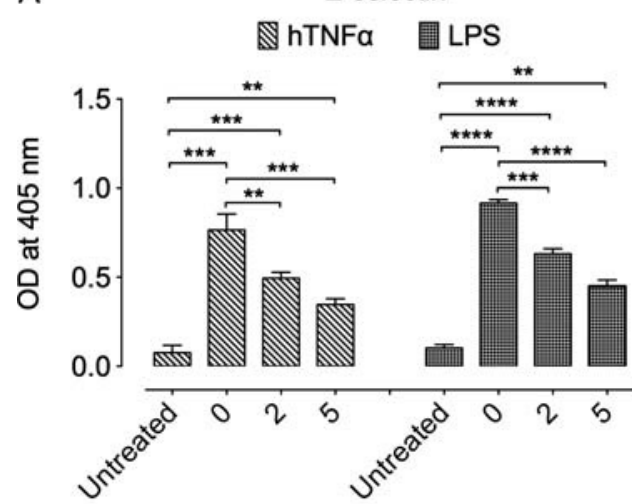

B

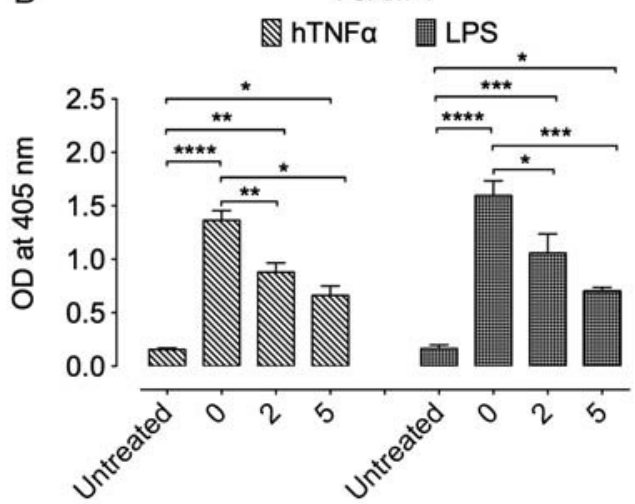

$\mathrm{Rb}$ anti-HMGB1 antibody $(\mu \mathrm{g} / \mathrm{ml})$

C

$\mathrm{Rb}$ anti-HMGB1 antibody $(\mu \mathrm{g} / \mathrm{ml})$

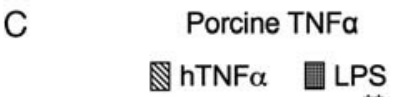

D

Porcine IL-8
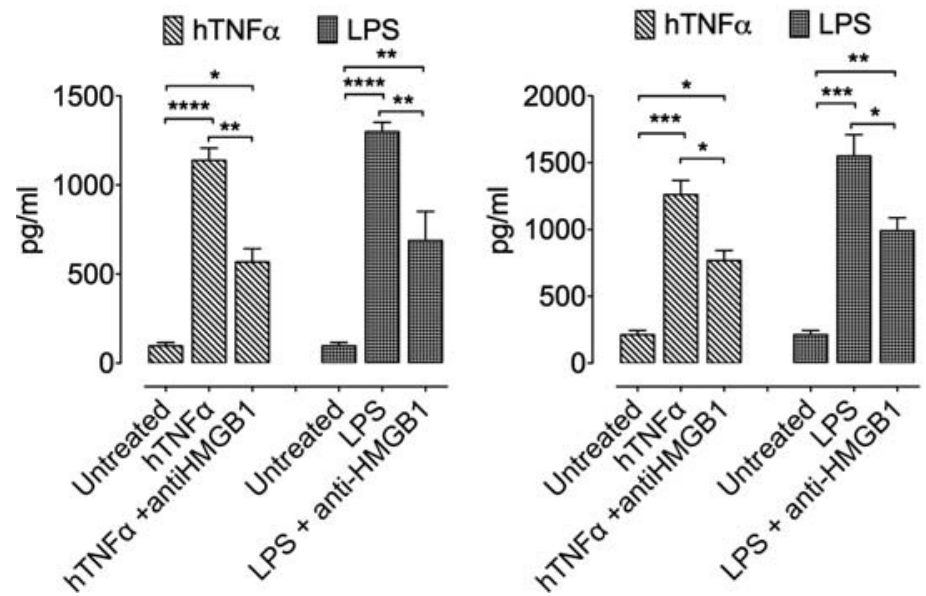

E

Porcine MCP-1

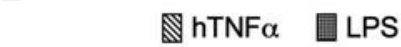

FIGURE 2. Effect of anti-HMGB1 antibody treatment on the hTNFa- and LPS-induced activation of WT PAEC. Confluent WT PAEC were treated with $\mathrm{hTNF} \alpha(10 \mathrm{ng} / \mathrm{mL})$ or LPS $(100 \mathrm{ng} / \mathrm{mL})$ in the presence of rabbit anti-HMGB1 antibody $(0-5 \mu \mathrm{g} / \mathrm{mL})$ for 16 hours and the cell surface expression of (A) E-selectin and (E) VCAM-1 was measured by a cell-based ELISA. (C-E) After 16 hours of treatment with hTNFa or LPS in the presence of anti-HMGB1, cell culture supernatants of WT PAEC were analyzed for the release of porcine proinflammatory cytokines (C) TNFa (D) IL-8 and (E) MCP-1 by using commercial ELISA kits. Statistical analysis was done by 1-way ANOVA testing with Bonferroni correction $\left({ }^{\star} P<0.05,{ }^{\star *} P<0.01,{ }^{* \star *} P<0.001,{ }^{\star \star \star \star} P<0.0001\right)$. All results are shown as mean $\pm \mathrm{SD}$ of 3 independent experiments.

was significantly lower in hTBM PAEC as compared with WT PAEC (Figures 3A-C). No additional protection from HMGB1or hTNF $\alpha$-induced upregulation of E-selectin, VCAM-1, and
ICAM-1 expression was observed when GTKO.hCD46.hTBM PAEC were used instead of hTBM-only PAEC (Figure S2, SDC, http://links.lww.com/TP/B262).
A

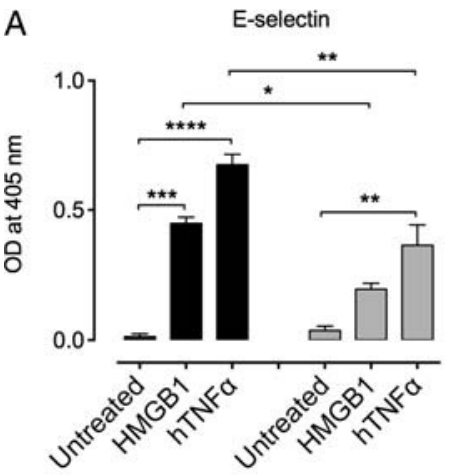

B

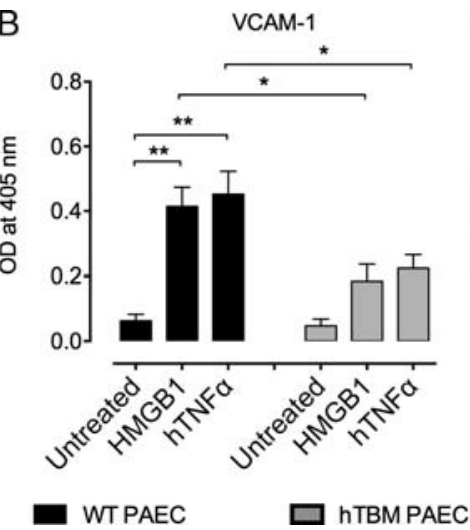

C

ICAM-1

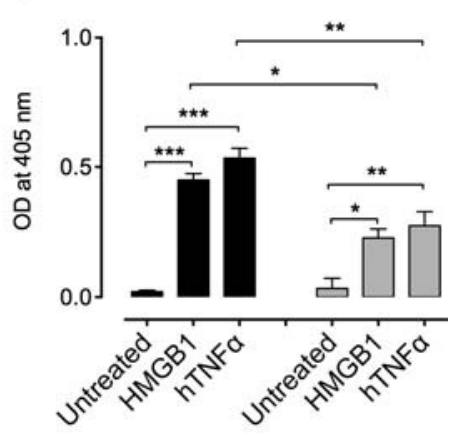

FIGURE 3. Effect of hTBM on the HMGB1- or hTNFa-mediated expression of cell adhesion molecules in PAEC. A-C, WT and hTBM PAEC were treated with HMGB1 (100 ng/mL) or hTNFa (10 ng/mL, positive control) for 16 hours, and the cell surface expression of (A) E-selectin, (B) ICAM-1 and (C) VCAM-1 was measured by cell ELISA. D-F, Significance was tested using 1-way analysis of variance with Bonferroni correction $\left({ }^{\star} P<0.05,{ }^{\star \star} P<0.01,{ }^{\star \star \star} P<0.001,{ }^{\star \star \star \star} P<0.0001\right)$. Data are mean $\pm \mathrm{SD}$ of 3 independent experiments. 


\section{HMGB1 Induces the Secretion of Proinflammatory Cytokines by PAEC}

To further examine the role of HMGB1 in proinflammatory cytokine release, WT and hTBM PAEC were incubated with HMGB1 or hTNF $\alpha$ as positive control, and the levels of secreted proinflammatory porcine cytokines TNF $\alpha$, IL-8, and MCP-1 were quantified by ELISA. Secretion of these cytokines was upregulated in both WT and hTBM PAEC. However, cytokine secretion by hTBM PAEC was significantly lower than by WT PAEC (Figure 4).

\section{HMGB1 Transforms PAEC to a Procoagulant and Antifibrinolytic Phenotype}

Wild-type and hTBM PAEC were stimulated with HMGB1 or hTNF $\alpha$ and expression of FGL2 and PAI-1 as procoagulant and antifibrinolytic markers, respectively, was evaluated by cell ELISA. Both FGL2 and PAI-1 were significantly upregulated on WT PAEC, whereas only the increase of PAI-1 was significant on hTBM PAEC (Figures 5A-B). For both surface markers, upregulation was reduced on hTBM as compared to WT PAEC.

In addition, the formation of PAI-1/tPA complexes was measured in culture supernatants obtained from WT and hTBM PAEC incubated with HMGB1 or hTNF $\alpha$. Baseline values of PAI-1/tPA complexes were minimal and not significantly different between WT and hTBM PAEC samples. Formation of PAI-1/tPA complexes significantly increased after stimulation of WT but not hTBM PAEC, and PAI-1/tPA complex formation was reduced in hTBM as compared with WT PAEC (Figure 5C).

\section{Transgenic hTBM Accelerates the Cleavage of HMGB1 by Thrombin}

To examine cleavage of HMGB1 by human thrombin bound to TBM, WT and hTBM PAEC were incubated with HMGB1 (10 $\mu \mathrm{g} / \mathrm{mL}$ for 30 minutes) with or without pretreatment of the cells with human thrombin $(5 \mathrm{U} / \mathrm{mL}$ for 30 minutes). Western blot/Image J analysis of supernatants showed that the presence of hTBM significantly enhanced thrombin-mediated cleavage of HMGB1 as compared to WT PAEC (Figures 6A-B).

Next, we examined the functional consequences of HMGB1 degradation by thrombin in the presence of hTBM. Wildtype and hTBM PAEC were stimulated with HMGB1
(100 ng/mL) or hTNF $\alpha$ (10 ng/mL) for 16 hours with or without pretreatment of the cells with human thrombin $(5 \mathrm{U} / \mathrm{mL})$ for 60 minutes. Expression of E-selectin and VCAM-1 was then measured by cell ELISA. Pretreatment of the cells with thrombin resulted in significantly downregulated expression of E-selectin and VCAM-1. In addition, the presence of the hTBM transgene led to a significant decrease of E-selectin and VCAM-1 expression as compared with WT PAEC (Figures 6C-F).

\section{The Lectin-like Domain of Thrombomodulin Is Important for Inhibition of HMGB1-Induced Inflammation}

To study the role of TBM's lectin-like domain in antiinflammatory responses, hTBM PAEC were treated with $10 \mu \mathrm{M}$ LPA for 10 minutes. Lysophosphatidic acid is known to cleave the lectin-like domain from TBM, and this was confirmed using the monoclonal anti-TBM antibody D-3, with specificity for the lectin-like domain. Antibody D-3 and clone 141C01, which is specific for the epidermal growth factor (EGF)-like domain of TBM, were used for immunofluorescence staining of LPA-treated PAEC. Complete absence of D-3 antibody binding indicated cleavage of the lectin-like domain of hTBM by LPA, whereas no difference for EGF-like domain staining with the 141C01 antibody was observed. In addition, expression of CD31, an endothelial cell marker, was not influenced by LPA treatment (Figures 7A-B).

To verify whether cleavage of the lectin-like domain influences the anti-inflammatory effect of TBM via binding of HMGB1, WT and hTBM PAEC were treated with HMGB1 or hTNF $\alpha$ for 16 hours with or without pretreating the cells with $5 \mu \mathrm{M}$ LPA for 10 minutes. Cleavage of the lectin-like domain by LPA resulted in significantly increased HMGB1- or hTNF $\alpha$-induced expression of E-selectin and VCAM-1 as compared to PAEC without LPA treatment (Figures 7C-F).

\section{DISCUSSION}

In this study, we have demonstrated that HMGB1 exerts potent proinflammatory and procoagulant effects on WT PAEC, and that hTBM-expressing PAEC are less sensitive to activation by either HMGB1 or hTNF $\alpha$. Expression of hTBM on PAEC inhibits hTNF $\alpha$ - or LPS-mediated secretion of HMGB1 and attenuates HMGB1-mediated responses,
A

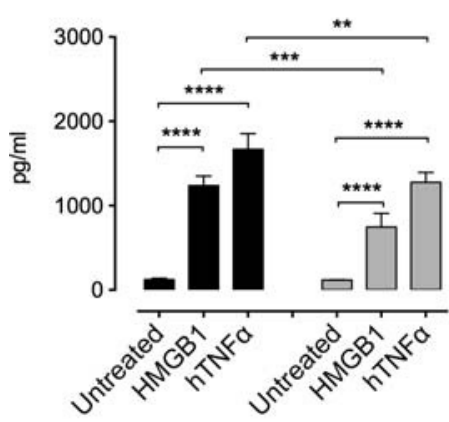

B

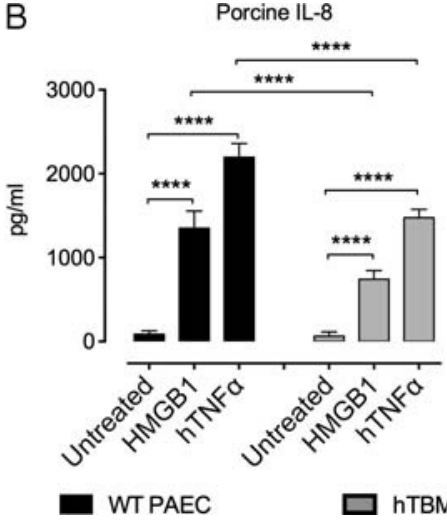

C
Porcine MCP-1

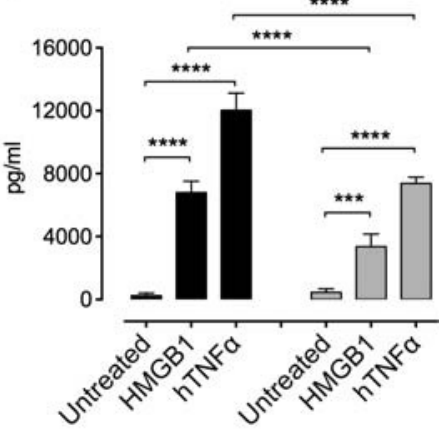

FIGURE 4. Effect of hTBM on the HMGB1- or hTNFa-mediated secretion of proinflammatory cytokines in PAEC. A-C, Cell culture supernatants of WT and hTBM PAEC treated with HMGB1 $(100 \mathrm{ng} / \mathrm{mL})$ or hTNFa $(10 \mathrm{ng} / \mathrm{mL})$ for 16 hours were measured for the levels of porcine proinflammatory cytokines (D) TNFa, (E) IL-8 and (F) MCP-1 by ELISA. Date are mean values \pm standard deviations of at least 3 independent experiments. Significance was tested using 1 -way ANOVA with Bonferroni correction $\left({ }^{\star} P<0.05,{ }^{\star \star} P<0.01,{ }^{\star \star \star} P<0.001,{ }^{\star \star \star \star} P<0.0001\right)$. 
A

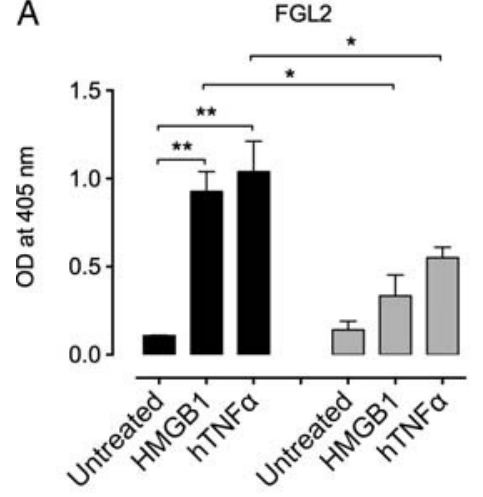

B

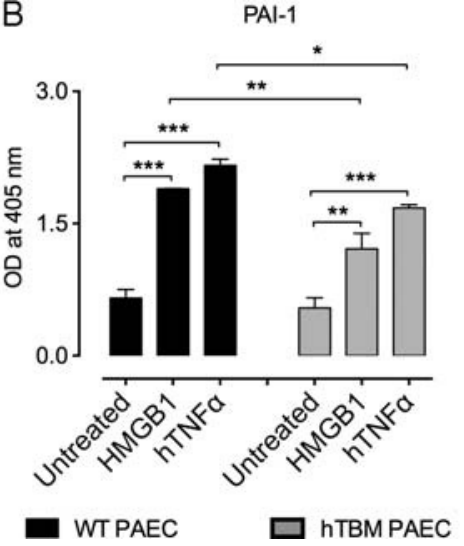

C

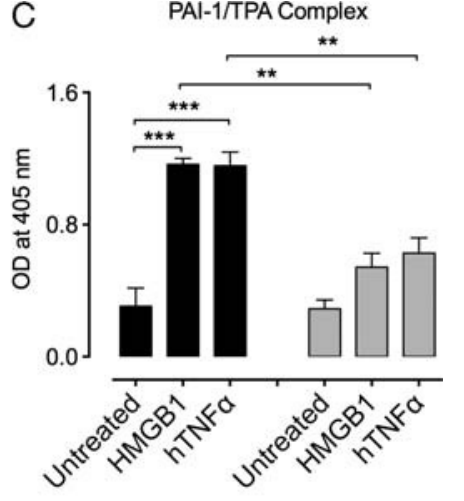

FIGURE 5. Inhibition of HMGB1 - or hTNFa-induced procoagulant and antifibrinolytic activities in PAEC by transgenic expression of hTBM. AB, WT and hTBM PAEC were treated with HMGB1 (100 ng/mL) or hTNFa (10 ng/mL, positive control) for 16 hours, and the cell surface expression of procoagulant FGL2 and (B) antifibrinolytic PAI-1 was measured by cell ELISA. C, Cell culture supernatants of PAEC treated as above were collected and processed for the levels of fluid-phase antifibrinolytic marker, PAl-1/tPA complex formation by ELISA as described in Materials and methods. Statistical analysis was carried out by 1-way ANOVA testing with Bonferroni correction. Data are mean \pm SD, ${ }^{\star} P<0.05,{ }^{\star \star} P<0.01,{ }^{\star \star \star} P<0.001,{ }^{\star \star \star \star} P<0.0001 ; n=3$ independent experiments.

presumably by sequestering HMGB1 and thus preventing binding to receptor for advanced glycation end-products and TLR2 and TLR4. The protection against the effects of LPS may have also been mediated in part by neutralization of the endotoxin by the lectin-like domain of TBM. ${ }^{5}$

High-mobility group box 1 has $99 \%$ sequence identity across mammalian species and is known to be involved in inflammatory responses. To test whether HMGB1 is secreted by PAEC and participates in endothelial activation, we stimulated WT PAEC with NHS or hTNF $\alpha$ or LPS. We showed that HMGB1 was secreted after stimulation of WT PAEC. It was shown recently that TBM plays an important role in suppressing inflammation, independent of its anticoagulant activity. ${ }^{15}$ In line with this finding, hTBM transgenic PAEC secreted less HMGB1 than WT PAEC upon stimulation by NHS, hTNF $\alpha$, or LPS. We also showed that HMGB1 stimulates WT PAEC to express the adhesion molecules E-selectin, VCAM-1, and ICAM-1 and to secrete the proinflammatory cytokines TNF $\alpha$, IL-8, and MCP-1. These findings indicate that HMGB1 activates porcine endothelial cells and that expression of hTBM can prevent this. High-mobility group box 1 also induced PAEC to express the procoagulant molecule FGL2 and to secrete the antifibrinolytic mediator PAI-1, indicating the induction of a procoagulant and an antifibrinolytic state of the endothelium. The latter was further supported by the increased formation of PAI-1/tPA complexes, which are fluid-phase antifibrinolytic markers. This transition of PAEC to a procoagulant and antifibrinolytic state, which triggers thrombotic microangiopathy in pig-toprimate xenotransplantation, ${ }^{1}$ was significantly inhibited by transgenic hTBM expression. Transgenic hTBM on the surface of endothelial cells binds and prevents dissemination of HMGB1, thereby reducing the engagement of HMGB1 with its proinflammatory receptors. ${ }^{16}$ Therefore, neutralization of HMGB1 by overexpression of hTBM represents a possible strategy to control HMGB1-mediated PAEC activation, preventing the establishment of a procoagulant and antifibrinolytic state of the porcine endothelium during xenograft injury.

Recombinant soluble hTBM is approved for clinical treatment of disseminated intravascular coagulation in
Japan, and has been shown to inhibit HMGB1 in several small animal models. Treatment with soluble hTBM might therefore be considered as an alternative to transgenic expression. However, soluble TBM appears to have less potent anti-inflammatory effects on PAEC than membranebound TBM. ${ }^{17}$ Furthermore, overexpression of hTBM on the xenograft vascular surface restricts anticoagulation and anti-inflammatory functions to where they are needed most. We therefore believe that, in the setting of xenotransplantation, the transgenic approach to boost TBM activity has considerable advantages over systemic treatment with soluble TBM.

We have previously reported that the lectin-like domains of porcine and human TBM share $71.4 \%$ amino acid identity. $^{2}$ The lectin-like domain of TBM is not involved in thrombin binding and has no known role in the activation of protein C. ${ }^{15}$ However, recent studies revealed that the lectin-like domain directly modulates inflammatory responses by scavenging HMGB1, which can then be cleaved and inactivated by TBM-bound thrombin. ${ }^{16,18}$ Our results with WT PAEC indicate that the lectin-like domain in porcine TBM can bind HMGB1 and promote, at least to some extent, its cleavage by human thrombin, and that overexpression of hTBM significantly accelerates this process. Although we were unable to directly compare the surface levels of porcine and human TBM due to the unavailability of an appropriate antibody, our results suggest that the more effective neutralization of HMGB1 by the transgenic PAEC is most likely due to increased expression of (total) TBM. Furthermore, hTBM PAEC showed reduced surface expression of E-selectin and VCAM-1 when pretreated with thrombin and then incubated with HMGB1. These findings are consistent with previous results, showing that thrombincleaved HMGB1 has significantly decreased proinflammatory activities. ${ }^{18}$

Lysophosphatidic acid, a bioactive lipid mediator present in biological fluids during endothelial damage or injury, enhances shedding of the lectin-like domain of TBM from human vascular endothelial cells. ${ }^{19}$ We treated hTBM PAEC with LPA and showed that LPA specifically cleaved the lectin-like domain of TBM, whereas its EGF-like domain 
A

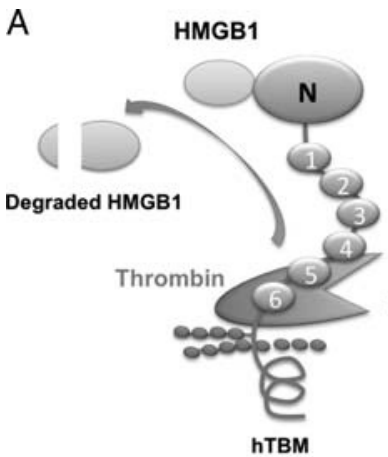

C

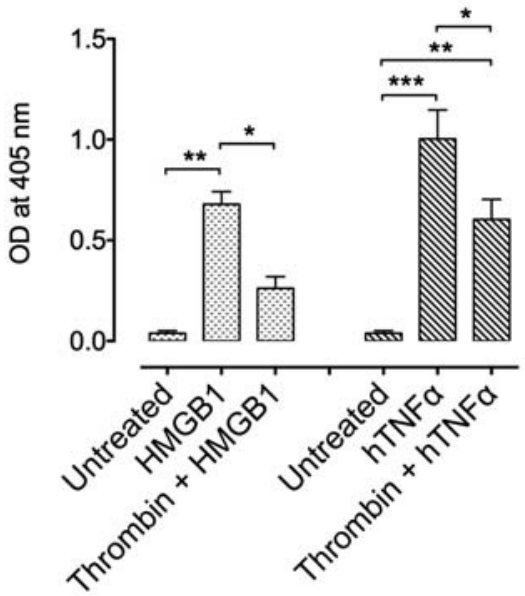

E

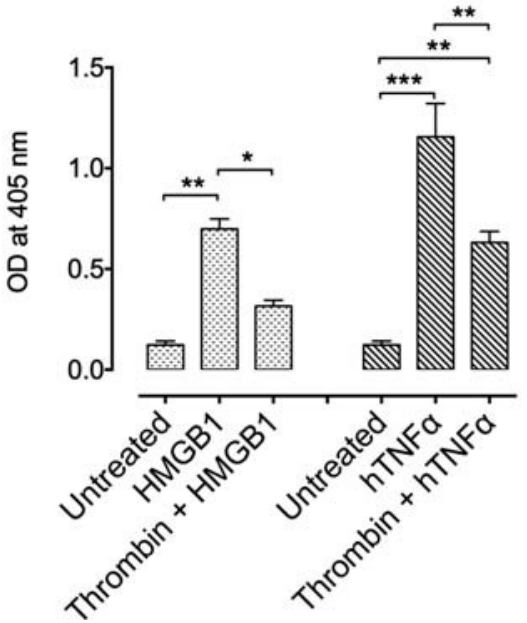

B

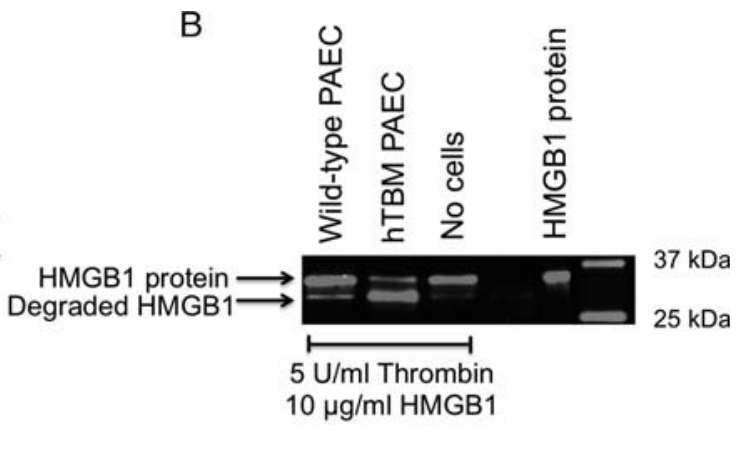

D

hTBM PAEC: E-selectin

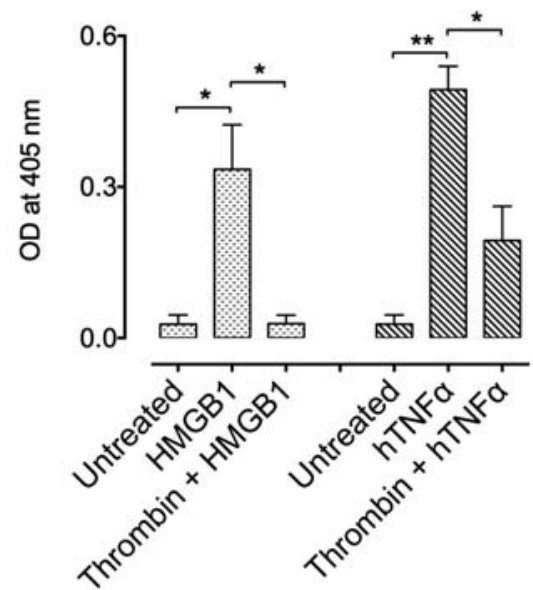

F

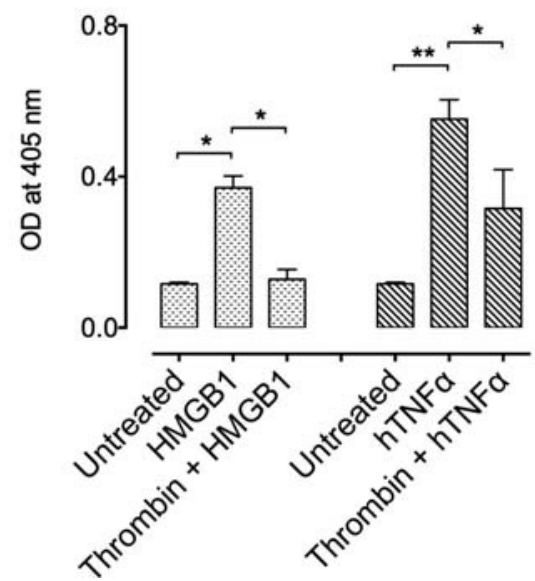

FIGURE 6. Transgenic hTBM enhanced thrombin-mediated proteolytic cleavage of HMGB1 to a less potent stimulus. A, Schematic representation of TBM's anti-inflammatory properties by sequestration and thrombin-mediated degradation of HMGB1. B, Western blot analysis of HMGB1 degradation. WT and hTBM PAEC were pretreated with human thrombin $(5 \mathrm{U} / \mathrm{mL})$ for 30 minutes before incubation with HMGB1 $(10 \mathrm{\mu g} / \mathrm{mL})$ for 30 minutes. Supernatants were subjected to SDS-PAGE followed by immunoblotting. One representative of 3 independent experiments is shown. After thrombin treatment for 60 minutes, ( $C$ and $\mathrm{E})$ WT and (D and F) hTBM PAEC were stimulated with HMGB1 (100 ng/mL) or hTNFa (10 ng/mL) for 16 hours, and the cell surface expression of (C-D) E-selectin and (E-F) VCAM-1 was measured by cell ELISA. Shown are mean values \pm standard deviations of at least 3 independent experiments. Significance was tested using 1-way ANOVA with Bonferroni correction $\left({ }^{\star} P<0.05,{ }^{\star \star} P<0.01,{ }^{\star \star \star} P<0.001\right)$.

stayed intact. Lysophosphatidic acid-induced TBM lectinlike domain shedding resulted in a significant enhancement of HMGB1- or hTNF $\alpha$-induced E-selectin and VCAM-1 expression, demonstrating the anti-inflammatory function of the lectin-like domain. Increased TBM expression on the transgenic PAEC reduced activation by HMGB1 or hTNF $\alpha$ compared with WT PAEC, and this protective effect was lost with LPA pretreatment. We conclude from these observations that the lectin-like domain of TBM indeed plays a role in regulating the proinflammatory action of HMGB1 on endothelial cells, which is further boosted by overexpression of hTBM on the transgenic PAEC. 
A

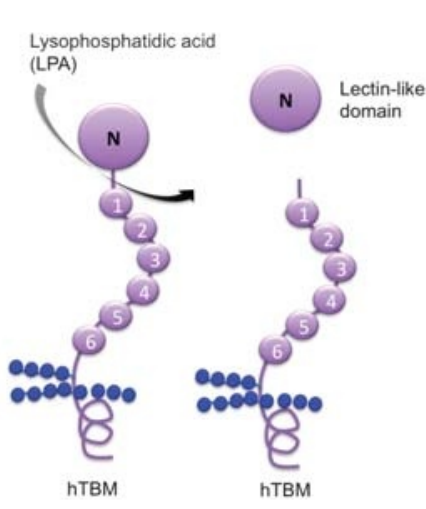

C

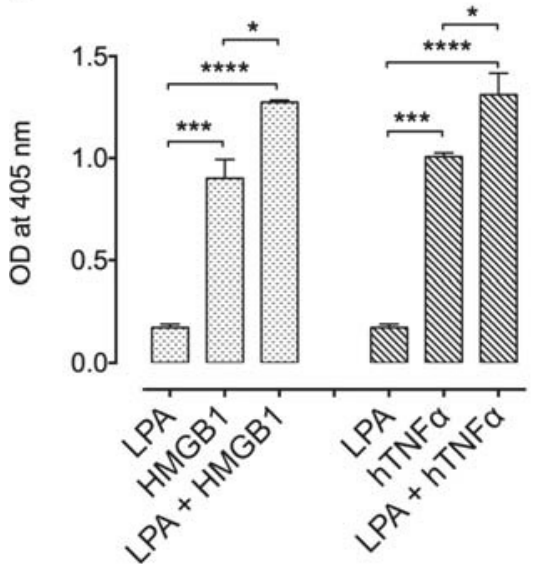

$E$

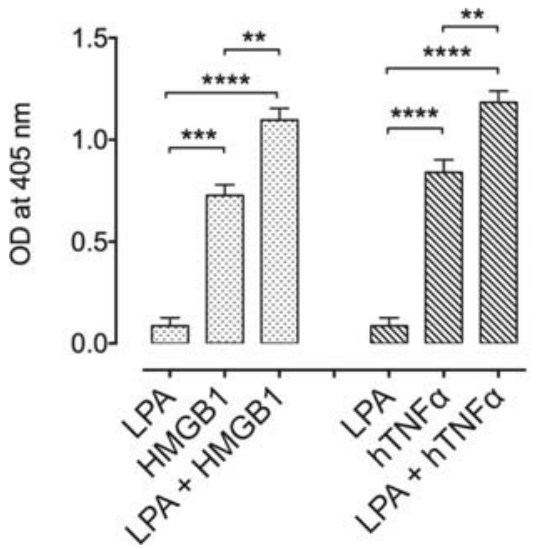

B Anti-hTBM Lectin-Domain Anti-hTBM EGF-Domain

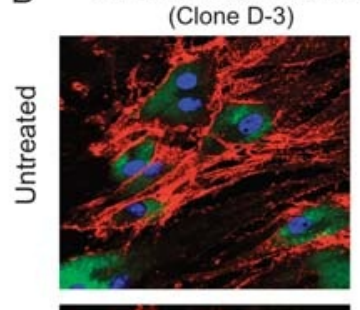

(Clone 141C01)
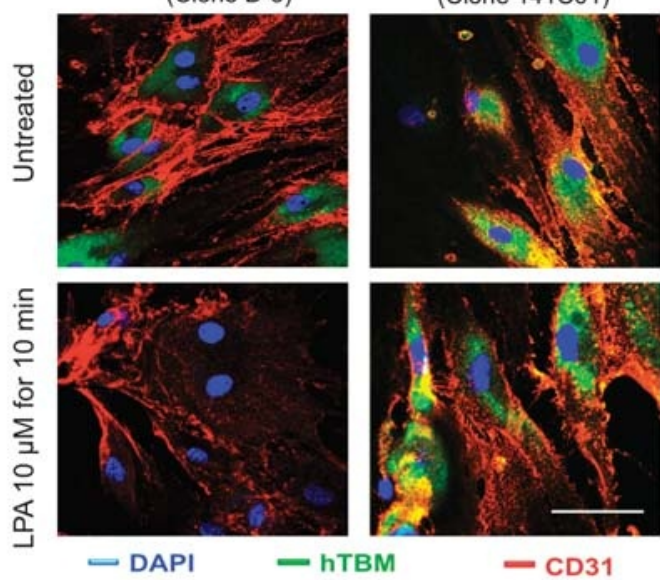

D

hTBM PAEC: E-selectin

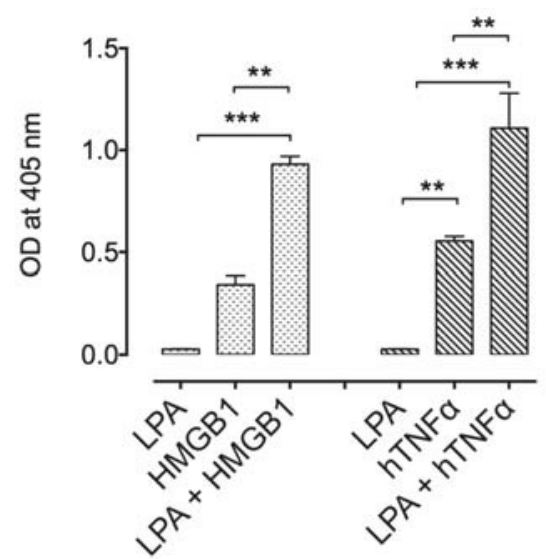

$\mathrm{F}$

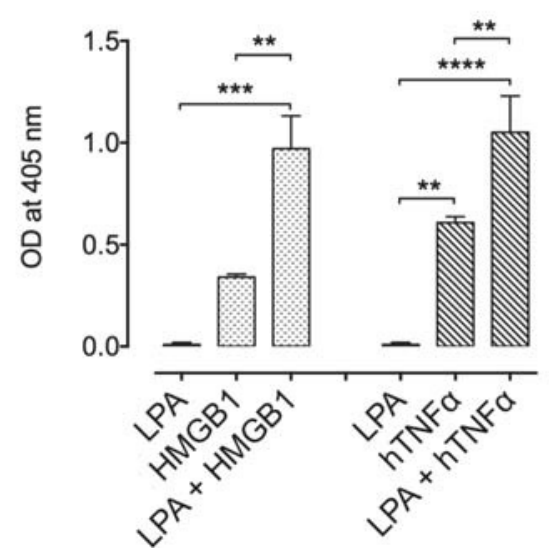

FIGURE 7. The lectin-like domain of hTBM provides protection against HMGB1- or hTNFa-induced PAEC activation. A, Schematic representation of LPA stimulated shedding of the lectin-like domain of hTBM. B, LPA-induced shedding of the lectin-like domain of TBM. Wild-type and hTBM PAEC were treated with $10 \mu \mathrm{M}$ LPA for 10 minutes, and analysed by immunofluorescence staining/confocal microscopy with an antilectin-like domain (clone D-3) or anti-EGF-like domain (clone 141C01) of the hTBM antibody and a rat anti-porcine CD31 antibody. Scale bar, $50 \mu \mathrm{m}$. C-F, Effect of LPA-mediated shedding of the lectin-like domain of hTBM on HMGB1- or hTNFa-induced PAEC activation. Wildtype and hTBM PAEC were pretreated with LPA $(10 \mu \mathrm{M})$ for 10 minutes before stimulation with HMGB1 (100 ng/mL) or hTNFa $(10 \mathrm{ng} / \mathrm{mL})$ for 16 hours, and analyzed for the expression of adhesion molecules ( $\mathrm{C}$ and E) E-selectin and (D and F) VCAM-1 by a cell-based ELISA. Statistical analysis was carried out by 1 -way ANOVA testing with Bonferroni correction. Data are mean $\pm \mathrm{SD},{ }^{\star} P<0.05,{ }^{* \star} P<0.01,{ }^{* \star *} P<0.001$, ${ }^{* * \star *} P<0.0001 ; n=3$ independent experiments. 
In conclusion, HMGB1 has profound proinflammatory, procoagulant and antifibrinolytic effects on endothelial cells. It upregulates the expression of adhesion molecules and transforms the endothelial cell surface to a procoagulant and antifibrinolytic state by expression of FGL2 and PAI-1. Transgenic expression of hTBM on PAEC limits these changes by binding HMGB1 and promoting its cleavage by thrombin.

\section{ACKNOWLEDGMENTS}

The authors thank Annegret Wuensch, Andrea Baehr from the Institute of Molecular Animal Breeding and Biotechnology, Ludwig-Maximilian University, Munich, Germany for providing aortic endothelial cells from genetically modified pigs. The authors also thank Evelyn Salvaris for expert technical support.

\section{REFERENCES}

1. Ekser B, Ezzelarab M, Hara H, et al. Clinical xenotransplantation: the next medical revolution? Lancet. 2012;379:672-683.

2. Roussel JC, Moran CJ, Salvaris EJ, et al. Pig thrombomodulin binds human thrombin but is a poor cofactor for activation of human protein $\mathrm{C}$ and TAFI. Am J Transplant. 2008;8:1101-1112.

3. Wuensch A, Baehr A, Bongoni AK, et al. Regulatory sequences of the porcine THBD gene facilitate endothelial-specific expression of bioactive human thrombomodulin in single- and multitransgenic pigs. Transplantation. 2014;97:138-147.

4. Iwase H, Ekser B, Satyananda V, et al. Pig-to-baboon heterotopic heart transplantation-exploratory preliminary experience with pigs transgenic for human thrombomodulin and comparison of three costimulation blockade-based regimens. Xenotransplantation. 2015;22:211-220.

5. Shi CS, Shi GY, Hsiao HM, et al. Lectin-like domain of thrombomodulin binds to its specific ligand Lewis $Y$ antigen and neutralizes lipopolysaccharide-induced inflammatory response. Blood. 2008;112: 3661-3670.
6. Ito T, Maruyama I. Thrombomodulin: protectorate God of the vasculature in thrombosis and inflammation. J Thromb Haemost. 2011;9(Suppl 1): 168-173.

7. Scaffidi P, Misteli T, Bianchi ME. Release of chromatin protein HMGB1 by necrotic cells triggers inflammation. Nature. 2002;418:191-195.

8. Yang H, Wang H, Czura CJ, et al. The cytokine activity of HMGB1. J Leukoc Biol. 2005;78:1-8.

9. Lotze MT, Tracey KJ. High-mobility group box 1 protein (HMGB1): nuclear weapon in the immune arsenal. Nat Rev Immunol. 2005;5:331-342.

10. Wang $\mathrm{H}$, Yang $\mathrm{H}$, Czura $\mathrm{CJ}$, et al. HMGB1 as a late mediator of lethal systemic inflammation. Am J Respir Crit Care Med. 2001;164(10 Pt 1): 1768-1773.

11. Musumeci D, Roviello GN, Montesarchio D. An overview on HMGB1 inhibitors as potential therapeutic agents in HMGB1-related pathologies. Pharmacol Ther. 2014;141:347-357.

12. Huang $\mathrm{Y}$, Yin $\mathrm{H}$, Han J, et al. Extracellular hmgb1 functions as an innate immune-mediator implicated in murine cardiac allograft acute rejection. Am J Transplant. 2007;7:799-808.

13. Li JH, Zhao B, Zhu XH, et al. Blockade of extracellular HMGB1 suppresses xenoreactive $\mathrm{B}$ cell responses and delays acute vascular xenogeneic rejection. Am J Transplant. 2015;15:2062-2074.

14. Banz Y, Cung T, Korchagina EY, et al. Endothelial cell protection and complement inhibition in xenotransplantation: a novel in vitro model using whole blood. Xenotransplantation. 2005;12:434-443.

15. Li YH, Kuo CH, Shi GY, et al. The role of thrombomodulin lectin-like domain in inflammation. J Biomed Sci. 2012;19:34.

16. Abeyama K, Stern DM, Ito Y, et al. The N-terminal domain of thrombomodulin sequesters high-mobility group-B1 protein, a novel antiinflammatory mechanism. J Clin Invest. 2005;115:1267-1274.

17. Miwa $Y$, Yazaki S, Iwamoto $M$, et al. Functional difference between membrane-bound and soluble human thrombomodulin. Transplantation. 2015;99:702-709.

18. Ito T, Kawahara K, Okamoto K, et al. Proteolytic cleavage of high mobility group box 1 protein by thrombin-thrombomodulin complexes. Arterioscler Thromb Vasc Biol. 2008;28:1825-1830.

19. Wu HL, Lin Cl, Huang YL, et al. Lysophosphatidic acid stimulates thrombomodulin lectin-like domain shedding in human endothelial cells. Biochem Biophys Res Commun. 2008;367:162-168. 